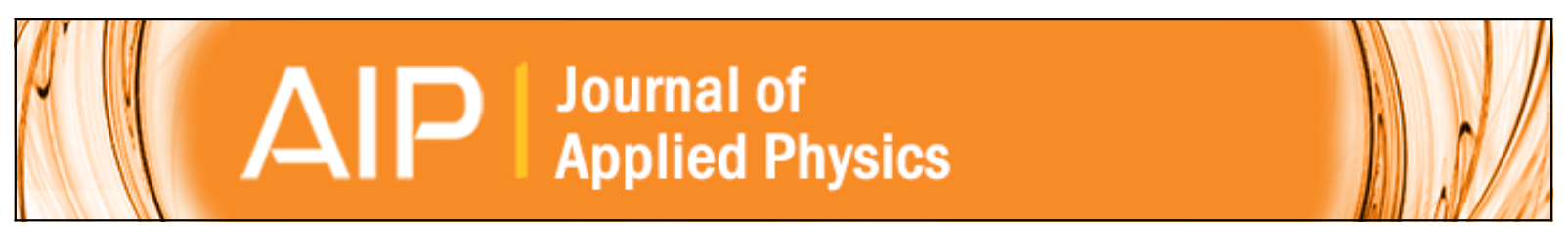

Quantum mechanical modeling of excited electronic states and their relationship to cathodoluminescence of BaZrO3

Mário L. Moreira, Juan Andrés, Lourdes Gracia, Armando Beltrán, Luciano A. Montoro, José A. Varela, and Elson Longo

Citation: Journal of Applied Physics 114, 043714 (2013); doi: 10.1063/1.4816247

View online: http://dx.doi.org/10.1063/1.4816247

View Table of Contents: http://scitation.aip.org/content/aip/journal/jap/114/4?ver=pdfcov

Published by the AIP Publishing

AlP 


\title{
Quantum mechanical modeling of excited electronic states and their relationship to cathodoluminescence of $\mathrm{BaZrO}_{3}$
}

\author{
Mário L. Moreira, ${ }^{1,2, a)}$ Juan Andrés, ${ }^{3}$ Lourdes Gracia, ${ }^{3}$ Armando Beltrán, ${ }^{3}$ \\ Luciano A. Montoro, ${ }^{4}$ José A. Varela, ${ }^{2}$ and Elson Longo ${ }^{2}$ \\ ${ }^{1}$ INCTMN, Physical and Mathematics Institute (IFM), Universidade Federal de Pelotas, \\ Campus Universitário Capão do Leão, s/n ${ }^{\circ}$. Caixa Postal 354, CEP 96010-900, Pelotas, RS, Brazil \\ ${ }^{2}$ INCTMN, Department of Physical Chemistry, Chemistry Institute, Unesp - Universidade Estadual Paulista, \\ Prof. Francisco Degni Street, s/n ${ }^{\circ}$, Quitandinha, Araraquara, SP 14800-900, Brazil \\ ${ }^{3}$ Departament de Química Física i Analítica, Universitat Jaume I, Campus del Riu Sec, Castelló E-12071, \\ Spain \\ ${ }^{4}$ LME, LNLS, Rua Giuseppe Máximo Scolfaro, 10.000 Pólo II de Alta Tecnologia, Campinas, SP, Brazil
}

(Received 4 September 2012; accepted 4 July 2013; published online 26 July 2013)

\begin{abstract}
First-principles calculations set the comprehension over performance of novel cathodoluminescence (CL) properties of $\mathrm{BaZrO}_{3}$ prepared through microwave-assisted hydrothermal. Ground (singlet, $s^{*}$ ) and excited (singlet $s^{* *}$ and triplet $t^{* *}$ ) electronic states were built from zirconium displacement of $0.2 \AA$ in $\{001\}$ direction. Each ground and excited states were characterized by the correlation of their corresponding geometry with electronic structures and Raman vibrational frequencies which were also identified experimentally. A kind of optical polarization switching was identified by the redistribution of $4 \mathrm{~d}_{\mathrm{z}} 2$ and $4 \mathrm{~d}_{\mathrm{xz}}(\mathrm{Zr})$ orbitals and $2 \mathrm{p}_{\mathrm{z}} \mathrm{O}$ orbital. As a consequence, asymmetric bending and stretching modes theoretically obtained reveal a direct dependence with their polyhedral intracluster and/or extracluster $\mathrm{ZrO}_{6}$ distortions with electronic structure. Then, $\mathrm{CL}$ of the as-synthesized $\mathrm{BaZrO}_{3}$ can be interpreted as a result of stable triplet excited states, which are able to trap electrons, delaying the emission process due to spin multiplicity changes. (C) 2013 AIP Publishing LLC. [http://dx.doi.org/10.1063/1.4816247]
\end{abstract}

\section{INTRODUCTION}

Understanding subtle processes which occur when electromagnetic radiation interacts with matter is still a great challenge. Both the mechanism that materials undergo after this irradiation and the optical properties derived from this interplay are of considerable importance in modern science and engineering. These responses depend on the intrinsic properties of the material and on the nature of the radiation. In the quantum electronic band theory, band formation requires a periodic potential, which is typically established by regularly spaced atoms in a crystal lattice. When this periodicity is perturbed by structural order-disorder effects, localized states may form in the band gap. ${ }^{1-4}$ Unlike extended states, localized states are able to confine charge carriers in space until they gain enough energy to escape. The excitation induced by electromagnetic radiation promotes electrons from the valence band $(\mathrm{VB})$ to the conduction band (CB); to probe the changes in the electronic and structural properties involved in the understanding of this mechanism, excited electronic states are a crucial component to understand optical processes in a broad range of fields which encompass physical-chemistry and materials science. Thus, an electronic excited state is obtained when a system (crystal) is in an energy level higher than the ground state; e.g., after absorption of one or more quanta of light (photons). However the behavior excited systems as well as

\footnotetext{
a) Author to whom correspondence should be addressed. Electronic addresses: mlucio@liec.ufscar.br and mlucio3001@gmail.com.Tel./Fax: +555332757614 .
}

the charge redistribution processes following excitation are not well understood, in part because studies on structural and electronic structures of these short-lived species are difficult to realize.

Without doubt, crystal and band structures of complex metal oxides are a key aspect in exploring new or improved functionalities. Traditional crystallographic structure determination involves finding atomic positions, lattice constants, and thermal parameters for all atoms in a crystal structure. However, often imperfections in crystal structures produce interesting chemical/physical properties. Currently, it is impossible to characterize the local ordering directly from diffraction data in such systems through conventional methods. Corresponding first-principles quantum mechanical calculations are essential to understand and interpret experimental results. In this sense, the characterization of ground and excited electronic states associated with optical behavior is vital to improve technological applications related to these properties. However, structural elucidation of these short-lived species has not been addressed due to considerable technical difficulties associated with measurements on time scales of microseconds or less. In addition, calculations of excited-state properties are still a challenge because they require the nontrivial task of an accurate determination of excited-state structures. ${ }^{5}$

Barium zirconate $\mathrm{BaZrO}_{3}$ (BZO) serves as an excellent model for a wide range of $\mathrm{ABO}_{3}$ perovskites which is relevant for numerous technological applications, such as high temperature material, electronic ceramics, non-linear optics, catalysis, superconductors, and so on. ${ }^{6-11}$ The current state 
of the art in the atomic structure solution of complex and disordered materials is density functional theory (DFT). DFT is clearly the first-principles method chosen for systems of this size because it offers good accuracy which sharply reduces the computation time and effort. ${ }^{12}$ Several calculations based on this method have been reported regarding the electronic and atomic structure of bulk BZO. ${ }^{13-21}$

A more complete understanding of the optical properties for perovskite-based materials is a prerequisite to eventual technological application, which is a key role for defects/ impurities and other structural order-disorder effects. ${ }^{7,22}$ Numerous efforts have been expended to understand the mechanism for optical emissions, particularly photoluminescence (PL) effects ${ }^{23-30}$ where device performance is strongly influenced by the energy and lifetime of the excited states. Borja-Urby et al. reported the photocatalytic activity for pure $\mathrm{BZO}$ and BZO bismuth doped. In both cases, the photocatalyst efficiency depends strongly of bismuth content as well induced defects. ${ }^{31}$ Recent studies have highlighted the important relationship of optical behavior and electronic excited states for different complex metal oxides such as $\mathrm{SrTiO}_{3}$ (Ref. 32) and $\mathrm{CaWO}_{4}{ }^{33}$ Theoretical characterization of the geometrical and electronic structures of ground and excited electronic states can complement experiments that are inherently limited by equipment acuracity; to the best of our knowledge, this approach has never before been attempted.

Among the different properties exhibited by the perovskite structure and currently under investigation, optical properties such as cathodoluminescence (CL) should be investigated. CL analysis is a mature technique which utilizes the light emitted from materials bombarded by a beam of high energy electrons. The produced spectra are a powerful method as well as a non-destructive way to investigate and characterize defects in the structure of solid materials. ${ }^{33}$ Very recently, Reddy et $a l .{ }^{34}$ reported a possible control over $\mathrm{CL}$ emission of $\mathrm{ZnO}$ supported on $\mathrm{CdSe}$ for nanostructured optoelectronic device applications. In addition, CL emission for nonstoichiometric silicon oxide has been reported at green and red regions of the visible spectrum due to nanoscaled tips and their coatings. 35

The goal of this article is to expand the theoretical understanding of $\mathrm{CL}$ phenomena in BZO using excited states. Although the physical and chemical behavior of this property was often mentioned and used in previous reports, it was always assumed to be a known prerequisite, and details about its appearance have not been completely investigated. DFT calculations were employed in the present research. A careful examination of calculated results provides an insight into the physical origin of this process which accounts for the CL behavior of BZO materials. The main goals of this paper can be summarized as follows: (1) the synthesis of BZO samples using the microwave-assisted hydrothermal (MAH) method; (2) the characterization of structural properties by XRD, Raman scattering and SEM (CL properties have been confirmed in the as-synthesized samples); (3) the localization and characterization of both ground and excited electronic states of BZO from firstprinciples calculations; and (4) the analysis of the geometry, electronic properties and vibrational frequencies of these electronic states to establish a relationship with CL emissions and structural order-disorder effects. Thus, the proposed mechanism of CL behavior is directly related to local and/or periodic imperfections (i.e., structural order-disorder effects) as they appear in both ground and excited electronic states. The remainder of the paper is organized as follows: Details of methods and model systems are given in Sec. II which is followed by an analysis and discussion of both theoretical and CL results in Secs. III and IV. The main conclusions are given in Sec. V.

\section{METHODS AND MODEL SYSTEMS}

First-principles calculations using DFT at the B3LYP level via self-consistent (SCF) method were carried out using the CRYSTAL09 package software. ${ }^{36}$ The B3LYP is composed of Becke's three-parameter hybrid non-local exchange functional $^{37}$ combined with the Lee-Yang-Parr gradient-corrected correlation functional, ${ }^{38}$ which has proven to be a very effective tool to deal with the present challenging problem. The atomic centers are described by all electron Gaussian basis sets for BZO: 976-31(621d)G for $\mathrm{Zr}$ and 6-31(d)G for $\mathrm{O}$ atoms (http://www.crystal.unito.it/Basis_Sets/Ptable.html website) and 976-311(631d)G for barium (http://www.tcm.phy.cam.ac. $\mathrm{uk} / \sim$ mdt26/basis_sets/Ba_basis.txt). The Gaussian basis set associated with the geometrical and respective keywords for the optimizations and parameterization is introduced via a typical CRYSTAL input formation, ${ }^{36}$ which can be found at CRYSTAL website (http://www.tcm.phy.cam.ac.uk/ mdt26/ basis_sets/Ba_basis.txt).

Parameters controlling the accuracy of the Coulomb and exchange integrals were set to $10^{-8}$ (ITOL1 to ITOL4) and $10^{-14}$ (ITOL5), whereas the percentage of Fock/Kohn-Sham matrix mixing was set to 40 (IPMIX $=40) .{ }^{36}$ The reciprocal space was sampled according to a regular sub-lattice determined by the shrinking factor ${ }^{36}$ which was set to 6 (13-20 independent $k$-points in the irreducible part of the Brillouin zone, depending on the dimensions of the adopted cell).

Periodic models based on the infinite translation of a repeat unit in three dimensions yield a physically correct solids picture. However, accurate excited electronic states calculation of periodic systems still represents a challenge for quantum-chemical methods. ${ }^{39}$ An excited state is obtained by imposing a low and high spin state that must promote an electron from the VB to the CB. Per unit cell, this transaction corresponds to imposing two electrons with the opposite (singlet) and same spin (triplet). It also creates a Frenkel exciton (hole in the VB, electron in the CB). We use periodic models to find the ground and excited electronic states. For singlet configurations in the input of optimized structure under $P_{4} / M M M$ and $P m \overline{3} m$ symmetry was used the option "spinlock" equal zero and for triplet configuration the "spinlock" was chosen as two.

In the BZO lattice, smaller Zr-site ions occupy cube corners in a six-fold oxygen coordination forming $\mathrm{ZrO}_{6}$ octahedra while larger Ba-site ions are positioned in the center of the cube in 12-fold coordination which forms the $\mathrm{BaO}_{12}$ cluster. Therefore, both $\mathrm{ZrO}_{6}$ and $\mathrm{BaO}_{12}$ clusters can be considered the polyhedra constituents of these systems, and they 
are arranged in a periodic pattern over long-range distances. Both the lattice parameters and internal atomic coordinates were used to perform the punctual calculations and for subsequent optimization to obtain fully relaxed structures which correspond to the equilibrium structure. The Mulliken charges were calculated using the local charge over each element into its respective cluster. To confirm the character of local minima on potential energy surfaces, vibrational frequency calculations were carried out to ensure that there are only positive frequencies which correspond to minima, for both ground and excited electronic states, and the following equations must be met: ${ }^{36}$

$$
\left(\frac{\partial E}{\partial x}\right)=0 \text { and }\left(\frac{\partial^{2} E}{\partial x^{2}}\right)>0 .
$$

To get the frequencies for cubic and pseudo-tetragonal cases of BZO excited states, the "FREQCALC" keyword was introduced immediately after the geometry configurations in the CRYSTAL input block. ${ }^{36}$

\section{EXPERIMENTAL SECTION}

\section{A. Material preparation and characterization}

The experimental procedure is as follows: (1) barium and zirconium chloride precursors were solubilized in doubly deionized water at $40^{\circ} \mathrm{C}$ to avoid carbonate formations. These solutions were mixed and co-precipitated by $6 \mathrm{M} \mathrm{KOH}$ and subsequently hydrothermalized at $140{ }^{\circ} \mathrm{C}$ for $160 \mathrm{~min}$ in a MAH system. The extended time $(160 \mathrm{~min})$ was chosen due to produce a better crystalline phase for the BZO system. ${ }^{40,41}$ After hydrothermalization the precipitate powder was centrifuged and washed until $\mathrm{pH}=7$ to eliminate the excess of mineralization, and the white powder obtained was dried for $12 \mathrm{~h}$ at $80^{\circ} \mathrm{C}$.

This powder was identified as a pure BZO compound by XRD using a Rigaku diffractometer DMax 2500PC using $\mathrm{Cu}$ $\mathrm{K}_{\alpha 1}(\lambda=1.5406 \AA)$ and $\mathrm{Cu} \mathrm{K}_{\alpha 2}(\lambda=1.54434 \AA)$ radiation, and FT-Raman spectra were recorded on a RFS/100/S Bruker spectrometer with a $\mathrm{Nd}$ :YAG laser which provided an excitation light at $1064 \mathrm{~nm}$ with a spectral resolution of $4 \mathrm{~cm}^{-1}$; its maximum output power was maintained at $85 \mathrm{~mW}$ in the frequency range of 100 up to $1000 \mathrm{~cm}^{-1}$. Scanning electron microscopes are used frequently for CL studies because enable spectra and spectral images can be acquired from small areas of bulk materials at high magnification and high resolution. SEM imaging was performed by using a ZEISS SUPRA field emission microscope at $15 \mathrm{kV}$. Typical in-lens secondary electron (SE) detection was combined with CL imaging microscopy by using a variable pressure secondary electron (VPSE) detector which is based on visible light detection. UV-visible (UV-vis) absorption was recorded with a Cary $5 \mathrm{G}$ spectrometer in total reflection mode by an integration sphere from a 200 to $800 \mathrm{~nm}$ region.

\section{RESULTS AND DISCUSSIONS}

The CL emission is preceded by an excitation process via electron beam of the scanning microscope; the corresponding
CL images are shown in Figure 1. In general, increasing the accelerating voltage and filament current will result in an increase of the CL intensity because more luminescence centers will be excited. The increasing of accelerating voltage extends the penetration depth of the electron into the sample body. The electron penetration depth can be estimated using an empirical formula: $\mathrm{L}(\AA)=250 \mathrm{AEn} /(\mathrm{Zn} / 2 \rho)$, where $\mathrm{n}=1.2 /\left(1-0.29 \log _{10} \mathrm{Z}\right), \mathrm{A}$ is the atomic or molecular weight of the material, $\rho$ is the bulk density, $\mathrm{Z}$ is the atomic number or the number of electrons per molecule in the case compounds, and $\mathrm{E}$ is the accelerating voltage $(\mathrm{kV})$ which results in a few nanometers of penetration depth for BZO. Thus, the CL property is directly correlated and favored by the reversed crystallization crystal growth as recently published ${ }^{40}$ and is predominant within a few nanometers from the surface.

CL images shown in Figure 2 were obtained as follows: Initially an electron beam is accelerated, and an image is collected by in-lens (SE) detection (see Figure 2). Then, the electron beam is taken off and a VPSE detector is set up to collect the CL image. An analysis of the results reveals that the CL behavior is a typical delayed process, ${ }^{42}$ which results in a decay mechanism that may involve different spin multiplicity and/or cross-transference between excited states. In both cases, the electronic excited states must be capable of trapping an excited electron during a certain time period before it returns to its ground state. ${ }^{42,43}$ The origin of this novel behavior may be related to impurity/defect states originating from oxygen deficiency and/or structural defects on the surface and/or into the bulk. These kinds of defects are very common in perovskite-based materials and imply that the crystallographic disorder degree is one essential factor

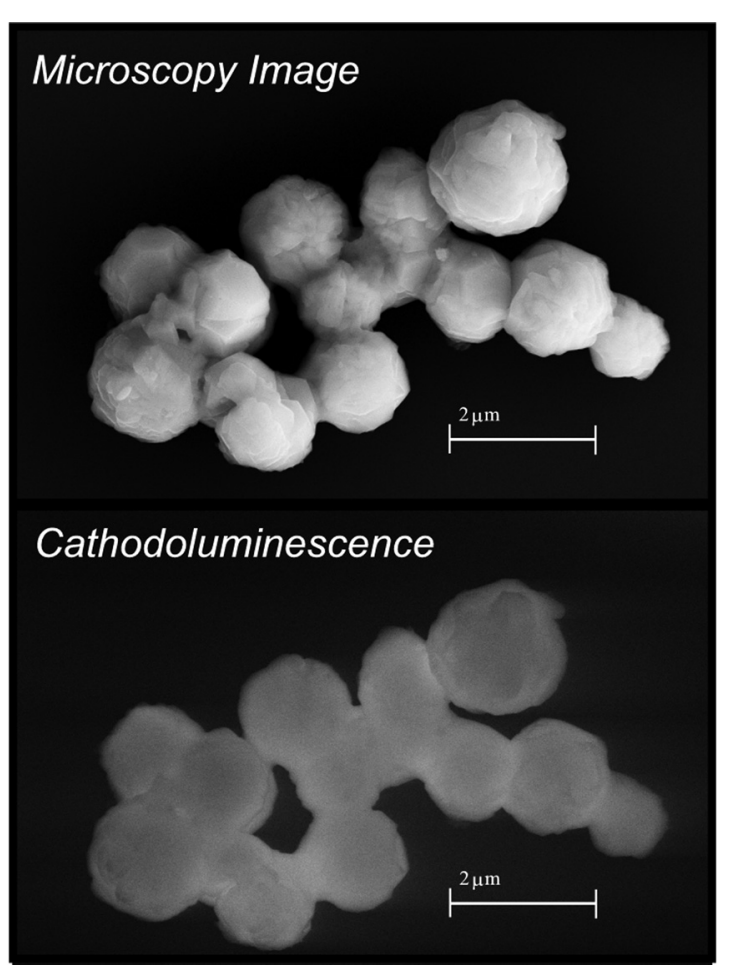

FIG. 1. FE-SEM image of BZO material (top) and its respective CL emission (below). 


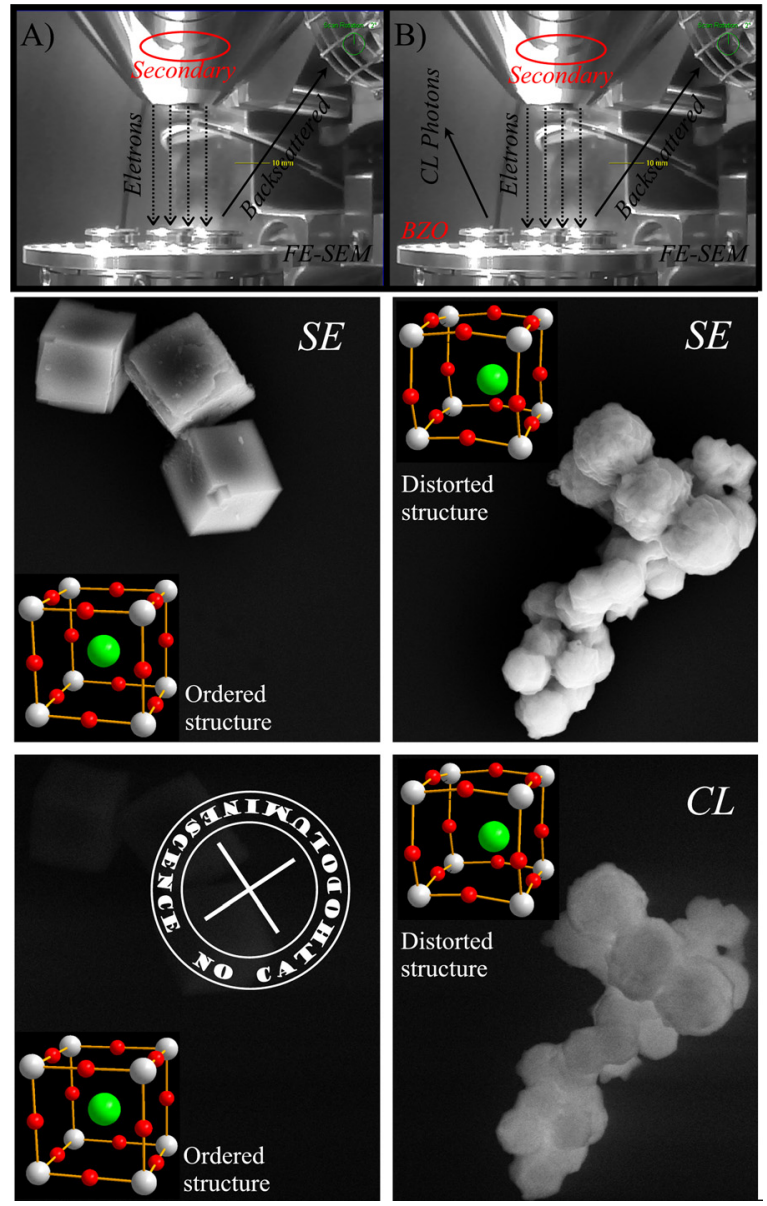

FIG. 2. Schematic design of CL measurement and its respective images. (A) The generic sample showing the FE-SEM image without the CL property; and (B) the FE-SEM image of the BZO sample and its CL image collected by a VPSE detector.

for the CL signal at room temperature. In addition, in Figure 2 the ordered and disordered structures are inserted for comparison, and these defects are uniformly distributed as indicated by CL images with no specific luminescent regions (see Figures 1 and 2). This material also delivers radioluminescence emission ${ }^{30}$ where the excitation source is $8 \mathrm{kV}$ which is slightly more than half the $15 \mathrm{kV}$ used for the CL excitation. The BZO also present a PL with an excitation of $3.5 \mathrm{eV}$. We postulate that our theoretical model can help to elucidate several aspects regarding this multiphotonic material.

To elucidate the luminescent process, first-principles calculations were carried out. The BZO ground state has a cubic structure, $P m \overline{3} m$. Then to find excited electronic states, different models have been used to perform a slight distortion on $\mathrm{ZrO}_{6}$ octahedra of cubic BZO Pm $\overline{3} m$ to obtain tetragonal symmetry in the space group $P_{4} / M M M$. This change in symmetry is the result of $\mathrm{Zr}$ atom displacement from the center of the $\mathrm{ZrO}_{6}$ oxygen octahedra. A spontaneous polarization arises from this asymmetrical structure resulting in the Jahn-Teller effect $^{44}$ and asymmetrical relaxation of the six nearest oxygen atoms surrounding the $\mathrm{Zr}$ atom.

The models were built by performing a slight distortion on the cubic BZO with triplet multiplicity to slightly change the cubic structure into a pseudo-cubic structure. For the initial structural configuration (cubic symmetry), the excited singlet $\left(s^{*}\right)$ and ground singlet $(s)$ configurations were optimized; in the second case, for pseudo-tetragonal optimized structure $P_{4} / M M M$, two excited singlet $\left(s^{* *}\right)$ and triplet $\left(t^{* *}\right)$ arrangements were obtained, and therefore, three excited states have been found: singlet $s^{*}$, singlet $s^{* *}$, and triplet $t^{* *}$. Among four models, the original model is based on the closed-shell cubic singlet configuration $s$ which maintains the symmetry. The second $s^{*}$ is based on the first optimization of $s$ as a singlet configuration with a displacement of $0.2 \AA$ in the $(001)$ direction inward of the octahedral cage which results in a cubic distorted symmetry. The third model $s^{* *}$ was built over the optimized $s^{*}$ open-shell model. The singlet state was also optimized and resulted in a complete restructuring of internal parameters to produce a pseudotetragonal structure. The triplet model $t^{* *}$ was developed based on the $s^{* *}$ singlet model which was again optimized. The unit cell was completely restructured to a triplet state. This new structural arrangement is solely a consequence of the triplet configuration which supports our initial model where a structural distortion results in a new electronic configuration. For each case, the optimized structure was analyzed, and the most important geometrical parameter values are reported in Table I. The relative energy of excited states $s^{*}, s^{* *}$ and $t^{* *}$ to the ground state $s$ are $0.74 \mathrm{eV}, 0.01 \mathrm{eV}$ and $6.79 \mathrm{eV}$, respectively.

An analysis of the results shows that the transformation from the fundamental $s$ to $\mathrm{s}^{*}$ and $\mathrm{s}^{* *}$ is produced by a slight distortion of $\mathrm{ZrO}_{6}$ clusters. The cubic form undergoes displacive transitions to lower symmetry with no bond breaking which involves the structural order-disorder effect in $\mathrm{ZrO}_{6}$ clusters; for the case of singlet $s^{* *}$, this change occurs on a very flat energy surface. For $t^{* *}$, the a parameter increases from $4.1676 \AA$ to $4.294 \AA$ whereas the c parameters is equal to $4.205 \AA$. The $\mathrm{BaO}_{12}$ and $\mathrm{ZrO}_{6}$ polyhedra in the triplet state have two different metal-oxygen distances; i.e., eight $\mathrm{Ba}-\mathrm{O}$ distances of $3.005 \AA$ and four of $3.036 \AA$ and two $\mathrm{Zr}-\mathrm{O}$ distances equal to $2.102 \AA$ and four equal to $2.147 \AA$. Thus, all distortions are originated from $\mathrm{ZrO}_{6}$ tilt and/or internal distortions. Zirconiun atom is centered over $\Gamma$-point at the center of octahedra then theoretical frequency mode arises from distortions over the $\Gamma$-point in response to different electronic singlet and triplet configurations. Therefore, the

TABLE I. Optimized lattice parameters and bond distances for the ground singlet ( $\mathrm{s})$ and excited singlet $\left(s^{*}, s^{* *}\right)$ and triplet $\left(t^{* *}\right)$ electronic states. The number of bonds for each distance is in parenthesis.

\begin{tabular}{|c|c|c|c|c|c|}
\hline & \multicolumn{2}{|c|}{$P m \overline{3} m$} & \multicolumn{2}{|c|}{$P 4 m m$} & \multirow[b]{2}{*}{ Exp } \\
\hline & $\mathrm{S}$ & $\mathrm{s}^{*}$ & $\mathrm{~s}^{* *}$ & $\mathrm{t}^{* *}$ & \\
\hline $\mathrm{a}$ & 4.1676 & 4.1676 & 4.1679 & 4.294 & 4.2063 \\
\hline $\mathrm{c}$ & & & 4.1674 & 4.205 & \\
\hline \multirow[t]{3}{*}{$\mathrm{Ba}-\mathrm{O}$} & 2.947 (12) & 2.947 (12) & $2.946(4)$ & $3.005(8)$ & $2.974(12)$ \\
\hline & & & $2.947(4)$ & $3.036(4)$ & \\
\hline & & & $2.948(4)$ & & \\
\hline \multirow[t]{3}{*}{$\mathrm{Zr}-\mathrm{O}$} & $2.084(6)$ & $1.884(1)$ & $2.082(1)$ & $2.102(2)$ & $2.103(6)$ \\
\hline & & 2.094 (4) & $2.084(4)$ & $2.147(4)$ & \\
\hline & & $2.284(1)$ & $2.086(1)$ & & \\
\hline
\end{tabular}



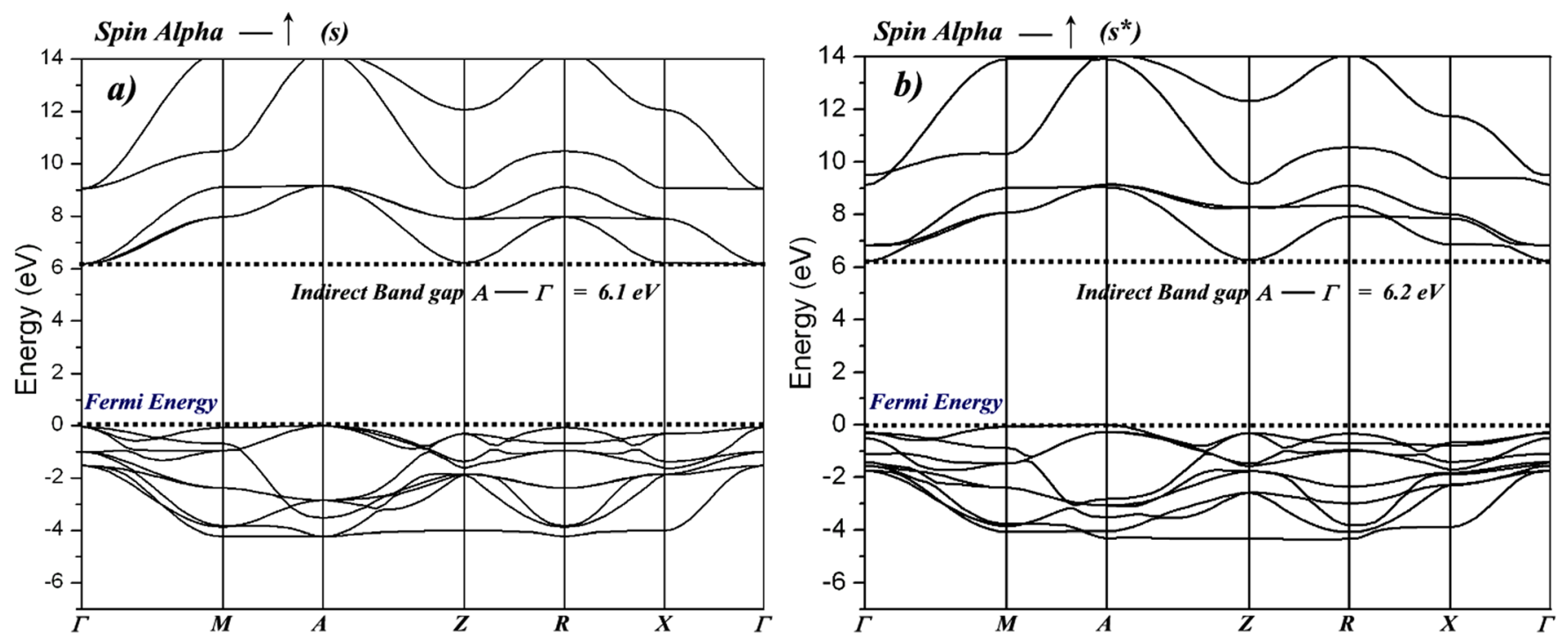

FIG. 3. Band structure for (a) ground " $s$ " and (b) " $s$ " electronic state; reporting the indirect A- $\Gamma$ transition.

structural order-disorder effect is an essential factor for the formation of excited states and the appearance of a CL signal at room temperature.

Mulliken charges reveal that the positive charge on the $\mathrm{Ba}$ atom, 1.81e, is preserved for all electronic states while the charge on the $\mathrm{Zr}$ atom decreases from $2.76 \mathrm{e}, 2.73 \mathrm{e}$ and 2.26e in $s, s^{*}$ and $t^{* *}$, respectively. To compensate this charge variation, the $\mathrm{O}$ atom negative charge decreases from $-1.53 \mathrm{e},-1.48 \mathrm{e}$ and $-1.30 \mathrm{e}$ (along the $c$ direction) in $s, s^{*}$ and $t^{* *}$, respectively. For the $\mathbf{s}^{* *}$ state, Mulliken charges are similar to the $\mathbf{s}$ state due to the small tetragonal distortion. The net charge value in terms of the $\left[\mathrm{ZrO}_{6}\right]$ cluster is $-1.81 \mathrm{e}$ in the $s$ state while the displacement of the $\mathrm{Zr}$ atom along the $c$ direction in the $s^{*}$ yields a distortion of $\left[\mathrm{ZrO}_{6}\right]$ clusters; these clusters present a net charge of $-0.33 \mathrm{e}$. This fact generates an imbalance of electronic density along the structural network to compensate for the enlargement/shortening of apical $\mathrm{Zr}-\mathrm{O}$ bonds. ${ }^{23,32}$ An analysis of the electronic spin density in the $t^{* *}$ state shows that the unpaired electrons are mainly localized on $\mathrm{Zr}$ atoms $(0.93 \mathrm{e})$ while a minor value is apparent on $\mathrm{O}$ atoms $(0.32 \mathrm{e}$ along $a$ and $b$ directions and $0.43 \mathrm{e}$ along the $c$ direction) as introduced above. Thus, the distortion between these complex clusters causes a polarization and/or a difference in the charge density which is able to promote a charge transfer process with the concomitant formation of hole and electron polarons.

A profound knowledge of optical spectroscopy is prominent from a basic point of view because optical features include not only the occupied and unoccupied parts of the electronic band structure but also the character of the bands. Therefore, the electronic structure of BZO was also investigated, and the total and projected densities of states are illustrated in Figures 5-7. Figures 3(a), 3(b), and 4 show the band structure of BZO obtained along the appropriate highsymmetry paths of the Brillouin zone for ground, $s$, and excited open-shell singlet, $s^{*}$, and triplet $t^{* *}$ electronic

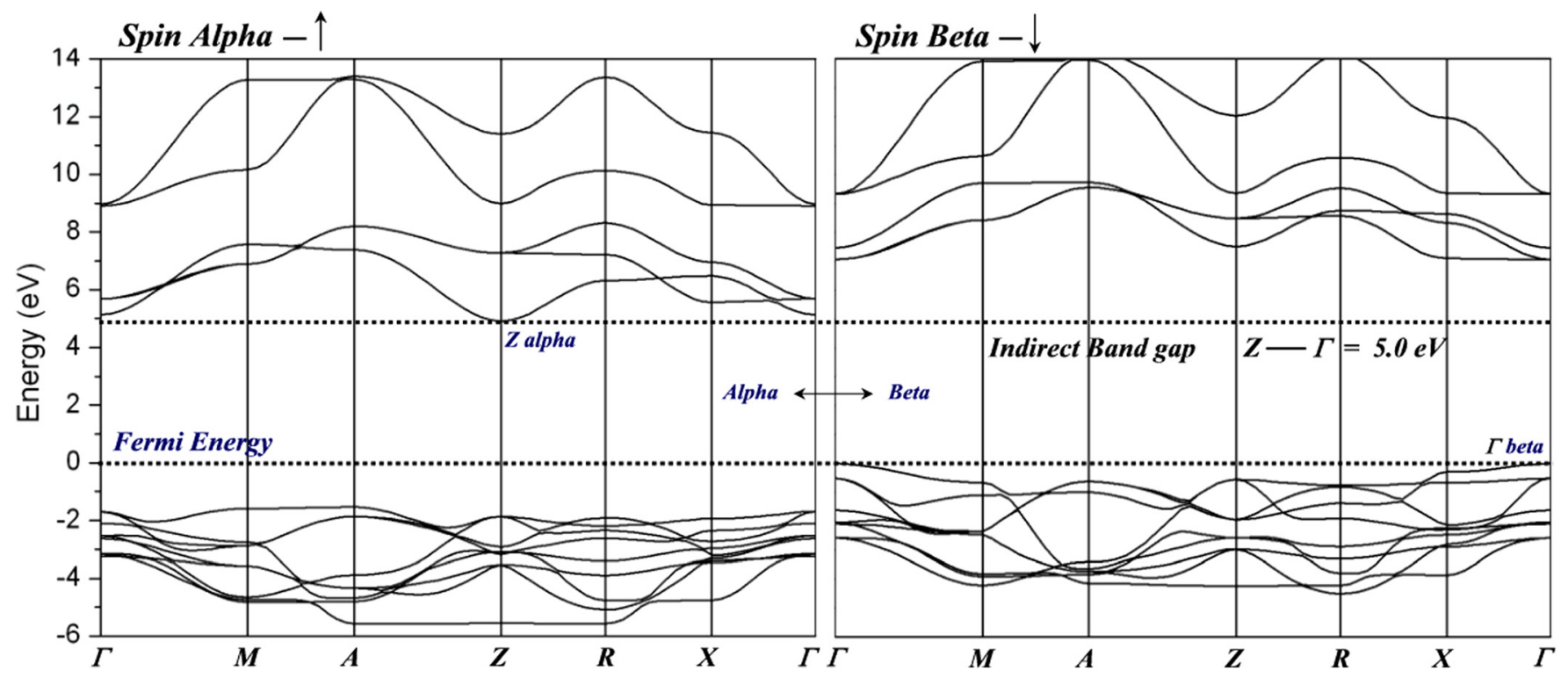

FIG. 4. Band structure of triplet state $t^{* *}$; reporting the indirect $\Gamma-\beta-Z-\alpha$ transition. 
states, respectively. In the first singlet state, despite the significant structural change, the band structure is only slightly affected. The electronic transition remains indirect with a slight increase in the band gap value. The most obvious changes are present between the k-points $\Gamma-\mathrm{M}$ and $\mathrm{R}-\Gamma$ for both conduction and valence bands. Moreover, for $t^{* *}$ the band structure is notably affected which produces a new indirect transition configuration from spin alpha to spin beta into different Brillouin zones for decay process. Furthermore, the band gap is strongly reduced from A$\Gamma=6.1 \mathrm{eV}$ for a singlet configuration to $\Gamma-\mathrm{Z}=5.0 \mathrm{eV}$ if a triplet state is considered. In Figure 4 the dotted lines are used to delimitate the valence and conduction bands, these lines touch the top of valence band at $0 \mathrm{eV}$ corresponding to $\Gamma$ point in the Brillouin zones as well as in the conduction band the dotted line touches the bottom of this band at $5 \mathrm{eV}$ corresponding to $\mathrm{Z}$ point. The same situation can be observed for singlet configuration, meanwhile the band gap is $6.1 \mathrm{eV}$ in this case. It is important to note that both $\alpha$ and $\beta$ electrons for the triplet configurations are separated in the Figure 4 to facilitate the visualization of their respective contributions, while they are superposed in the band structure.

An important observation is that experimental band gaps of BZO samples are around 4.8 to $5.1 \mathrm{eV} .^{31}$ The direct formation of a triplet as a result of a photon is a very improbable process, since both the electron spin and the orbit would need to change simultaneously. Thus, a singlet state is generally first formed by the absorption of a specific photon. Moreover, quite often the lifetime of one singlet state is too long for the spin of one of these electrons to be changed and thereby produce a triplet state. Thus, the triplet excited state is one possible model to represent the radiative luminescent process.

Direct observations of triplet band structure take to a conclusion that $\beta$ electrons are the main responsible by the top of valence band formation and the $\alpha$ electrons by the bottom of conduction band corresponding to $2 \mathrm{p}$ oxygen and zirconium $4 \mathrm{~d}$ contributions, respectively. These assumptions can be seen in Figure 7 for BZO triplet density of states (DOS).

For both cubic and distorted BZO in the singlet state, Figures 5 and 6 show that upper VBs correspond mainly to

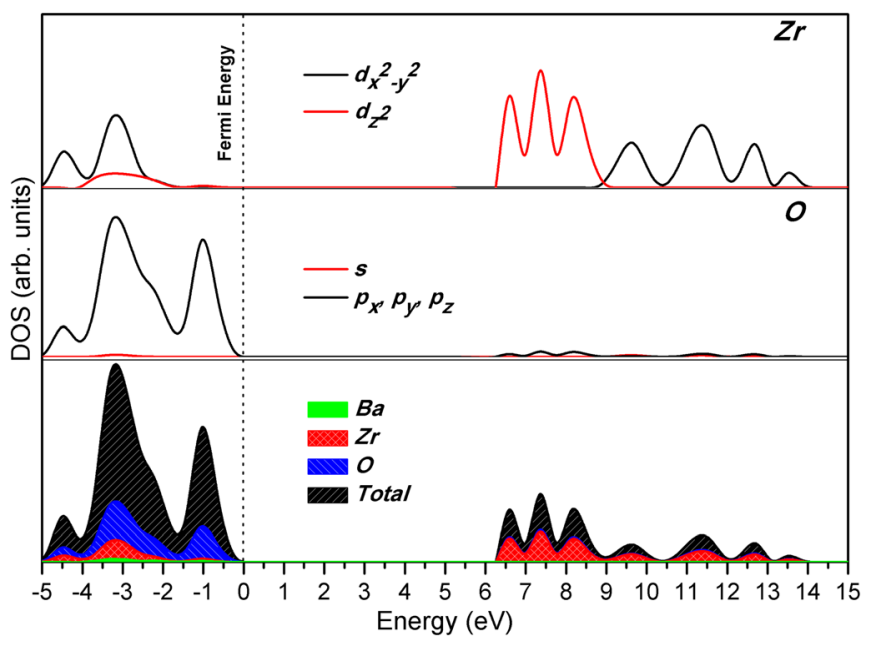

FIG. 5. DOS for cubic BZO at ground state $s$ of BZO.

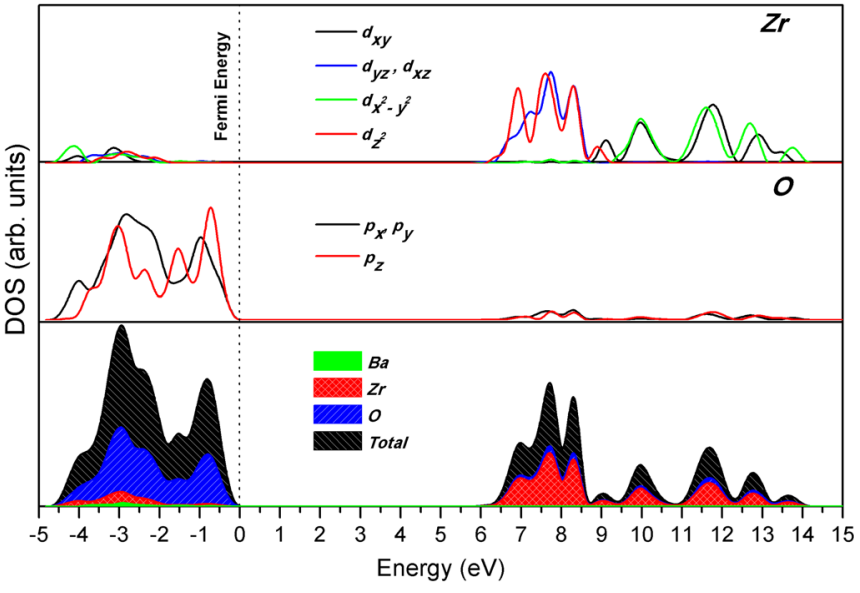

FIG. 6. DOS for the singlet state $s^{*}$.

$2 p(O)$ orbitals and receive a weak contribution from $4 d(\mathrm{dr})$ states; bottom CBs consist mainly of $4 \mathrm{~d}(\mathrm{Zr})$ orbitals with a slight contribution from $2 p(O)$ orbitals. The $4 \mathrm{~d}_{\mathrm{z}^{2}}(\mathrm{Zr})$ orbital dominates the bottom of the CB for cubic BZO $s$ whereas $4 d_{y z}$ and $4 d_{x z}$ also contribute to the bottom CB of the distorted structure $s^{*}$. Notice that the emergence of $4 \mathrm{~d}_{\mathrm{yz}}$ and $4 \mathrm{~d}_{\mathrm{xz}}$ participation in the $s^{*}$ model can be attributed mainly to $0.2 \AA$ displacement in the inward $\{001\}$ direction of the octahedral cage over $\Gamma$-point. Furthermore, the DOS shape is significantly changed in the $\mathrm{CB}$ region which indicates that the excited singlet configuration applied over the distorted structure generates a recombination of eigenfunctions in the overall structure.

The band structure analysis for the triplet state and its corresponding DOS illustrated in Figure 7 shows that the top VB of alpha electrons is constituted mainly of $2 p_{x}$ and $2 p_{y}$ oxygen orbitals located $1.5 \mathrm{eV}$ below the corresponding Fermi energy of beta electrons, where the $2 p_{z}(O)$ orbital contribution is dominant. Thus, the DOS shape in the VB

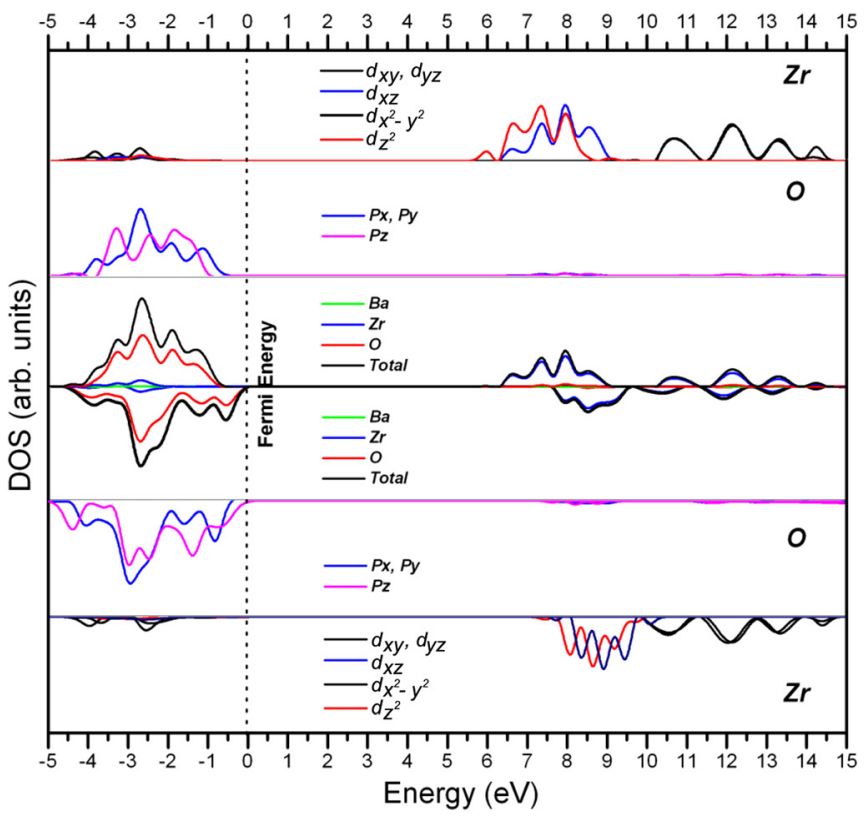

FIG. 7. DOS for the triplet electronic state $t * *$. The first three images refer to $\alpha$ spin electrons, and the balance refers to $\beta$ spin electrons. 
TABLE II. Vibrational modes in $\left(\mathrm{cm}^{-1}\right)$ for ground (s) and excited $\left(s^{*}, s^{* *}\right.$, and $\left.t^{* *}\right)$ electronic states of a BZO compound. Exp corresponds to experimental values. R, active Raman; IR, active infrared.

\begin{tabular}{|c|c|c|c|c|}
\hline \multirow[b]{2}{*}{$\operatorname{Exp}(\mathrm{R})$} & \multicolumn{2}{|c|}{$P m \overline{3} m$} & \multicolumn{2}{|c|}{$P 4 m m$} \\
\hline & $\mathrm{s}$ & $\mathrm{s}^{*}$ & $\mathrm{~s}^{* * *}$ & $t^{* *}$ \\
\hline 110 & $141.8(\mathrm{~F} 1 \mathrm{u}) \mathrm{IR}$ & 110.7 (A) R/IR & 112.0 (A) R/IR & 23.5 (A) R/IR \\
\hline 133 & & 112.7 (E) R/IR & 112.1 (E) R/IR & 69.1 (E) R/IR \\
\hline 153 & & & & 159.1 (E) R/IR \\
\hline 184 & & 215.6 (E) R/IR & 227.8 (A) R/IR & 171.8 (B) $\mathrm{R}$ \\
\hline 224 & & 244.2 (E) R/IR & 228.7 (E) R/IR & \\
\hline \multirow[t]{3}{*}{275,305} & $271.9(\mathrm{~F} 2 \mathrm{u}) \mathrm{R}$ & & 236.3 (E) R/IR & 252.6 (A) R/IR \\
\hline & & 258.1 (B) $\mathrm{R}$ & 236.7 (B) $\mathrm{R}$ & \\
\hline & 280.9 (F1u) IR & 348.5 (A) R/IR & & 297.3 (E) R/IR \\
\hline \multicolumn{5}{|l|}{$405,454,487$} \\
\hline \multirow[t]{2}{*}{$553,615,686$} & $635.2(\mathrm{~F} 1 \mathrm{u}) \mathrm{IR}$ & 586.7 (E) R/IR & 590.7 (E) R/IR & 639.0 (A) R/IR \\
\hline & & & 591.0 (A) R/IR & 652.2 (E) R/IR \\
\hline 731 & & 762.0(A) R/IR & & \\
\hline
\end{tabular}

and the $\mathrm{CB}$ is completely distinguished when compared with the singlet configuration. The first three $\mathrm{CB}$ bands of alpha electrons between 5 and $8.5 \mathrm{eV}$ confirm a great dispersion; the $4 \mathrm{~d}_{\mathrm{z}^{2}}(\mathrm{Zr})$ state is dominant in the first band, and the second and third bands present also an important $4 \mathrm{~d}_{\mathrm{xz}}(\mathrm{Zr})$ contribution. This fact can be associated with the shortening $(\sim 0.05 \AA)$ of two $\mathrm{Zr}-\mathrm{O}$ bonds along the $c$ direction as a result of c-compression with respect to the other four $\mathrm{Zr}-\mathrm{O}$ bonds of the $\mathrm{ZrO}_{6}$ octahedra as illustrated in Table I. The lower part of the $\beta$ electron (spin-down) CB is also composed of three bands with almost the same contributions, but they are located $2 \mathrm{eV}$ above the corresponding $\alpha$ (spin-up) bands.

The $\alpha-\beta$ transition for the triplet state is allowed for an emission along spin inversion process but is forbidden for absorptions. So we propose a model to elucidate the CL behavior. $\alpha$ electrons are excited from the VB to the $\mathrm{CB}$, and then they can change to a $\beta$ excited spin via crosstransference between singlet and triplet excited states, where were trapped for a certain time and then decay to a beta VB which emits photons in visible range of the spectrum. The ground state of barium zirconate was already characterized by Bilic and Gale $^{13}$ using three different methodologies based on plane waves with different functionals as LDA and GGA. In this work, the authors indicate an unstable cubic structure of barium zirconate underground state configurations. Recently, a similar methodology calculation displacing zirconium atom from the centrosymmetric position was applied to simulate local and periodic distortions of barium zirconate cubic symmetry. ${ }^{45}$ However, in that case any excited states were considered.

Raman spectroscopy is one of the most suitable methods for investigating and characterization of structure and symmetry in solids, particularly the degree of structural order at short range. ${ }^{46}$ Experimental and calculated vibrational modes for ground and excited electronic states are listed in Table II. An analysis of the results shows in Figure 8, reports that BZO obtained samples have active Raman scattering which indicates that the cubic symmetry is distributed and polarized at short range and is mainly due to distorted $\mathrm{ZrO}_{6}$ clusters. The calculated results show that experimentally observed absorption peaks can be assigned to modes obtained by the calculation. Depending on the crystal structure symmetry, some vibrational modes are allowed in the infrared absorption but forbidden in Raman scattering, and some vibrational modes in both processes are allowed or forbidden. In this study, the interpretation of the Raman active vibrational modes is reported which enables a more comprehensive understanding of BZO lattice vibrations along with the previous reports. ${ }^{13}$ Raman scattering for this king of systems are typically observed at low wave number range (in the range of $23-761 \mathrm{~cm}^{-1}$ ). The evolution of Raman spectra provides interesting changes. The first experimental mode at $101 \mathrm{~cm}^{-1}$ can be related to a soft mode and does not necessarily have a direct correlation with structural features, although the calculated modes reveal a lattice vibration associated with the $\mathrm{Zr}$ $\mathrm{Ba}-\mathrm{Zr}$ chain. The normal mode is associated with the distortion of $\mathrm{ZrO}_{6}$ octahedra in the cubic symmetry by the displacement of $\mathrm{Zr}$ atoms along the $z$ direction. A connection of $s$

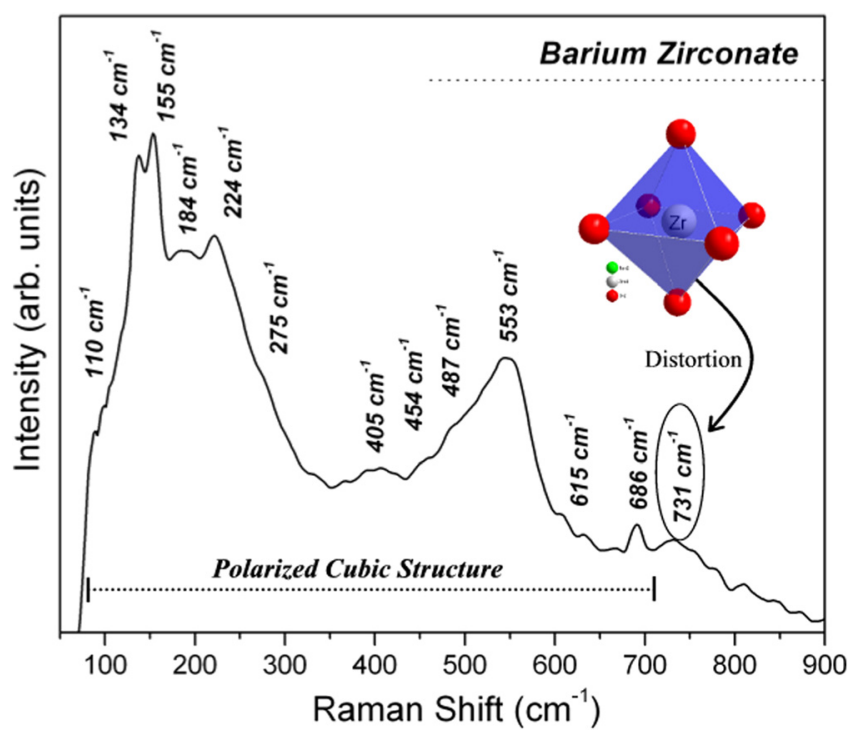

FIG. 8. First-order Raman active modes spectrum for cubic BZO considered as a natural excited state at room temperature. 
with $s^{*}$ electronic states has a value of $281 \mathrm{~cm}^{-1}$ and $348 \mathrm{~cm}^{-1}$ for modes $F_{1 u}$ and $A$, respectively. These modes correspond to the asymmetric bending $(\mathrm{O}-\mathrm{Zr}-\mathrm{O})$ where outer $\mathrm{O}$ and $\mathrm{Zr}$ atoms move in opposite directions. $s$ and $\boldsymbol{s}^{*}$ are also connected by stretching modes $F_{1 u}$ and $A$ with values of $635 \mathrm{~cm}^{-1}$ and $762 \mathrm{~cm}^{-1}$, respectively, which are related to the asymmetric $\mathrm{O}-\mathrm{Zr}$ stretching of central $\mathrm{O}$ atoms with regard to outer $\mathrm{O}$ atoms. Local and dynamic symmetries of Raman spectra for BZO also show a weak mode at $731 \mathrm{~cm}^{-1}$ (Ref. 47) which could be due to the different coherence length.

The normal mode associated with the structural distortion related to the transformation from cubic to tetragonal arrangement along the $z$ direction has a value of $635 \mathrm{~cm}^{-1}$ $\left(F_{1 u}\right), 591 \mathrm{~cm}^{-1}(A)$ and $639 \mathrm{~cm}^{-1}(A)$ for $s, s^{* *}$ and $t^{* *}$, respectively. In the $\mathbf{s}$ state, $\mathrm{Zr}$ atoms move along three crystal directions while in $\boldsymbol{s}^{* *}$ and $\boldsymbol{t}^{* *}$ states, $\mathrm{Zr}$ atoms stretch asymmetrically with regard to two neighboring $\mathrm{O}$ atoms along the $z$ direction. On the basis of the above results, we suggest that the $\mathrm{CL}$ in the undoped $\mathrm{BZO}$ results from excited electronic states as a consequence of the distortion of $\mathrm{ZrO}_{6}$ octahedra over the $\Gamma$-point and $\mathrm{BaO}_{12}$ cube-octahedral clusters. This local distortion not only modifies $\mathrm{Zr}-\mathrm{O}$ bond lengths but also induces noticeable changes in the structure and thereby alters the interaction among the electronic distributions on atom cells. These distortions are local; i.e., they involve several lattice constants and several vibrational periods.

Optical properties such as CL are strongly dependent upon the structural and morphological features of the crystal system. ${ }^{48}$ Structural order-disorder associated effects are a crucial factor in determining the optical behavior of material. ${ }^{49}$ Six-fold $\mathrm{ZrO}_{6}$ and 12 -fold $\mathrm{BaO}_{12}$ clusters are structural motifs of BZO. ${ }^{41}$ During the CL process, a symmetry breaking occurs at short order, which involves two clusters; i.e., structural distortions associated with the imperfect coordination mainly related to $\mathrm{Zr}$ sites. Then, the full coordination of the material disappears and the undercoordination associated with distorted $\mathrm{ZrO}_{6}$ and $\mathrm{BaO}_{12}$ clusters appears and yields the electronic excited state. This symmetry breaking is associated with order-disorder effects and is a necessary condition for the CL emission in the BZO system (see Figure 2). These structural changes can be related to alter of polarization between distorted clusters that are capable of populating stable excited electronic states and hence active vibrational Raman modes. The localization and characterization of excited states that can be considered as trap states implicated in this process are central to the observation of new CL phenomena in BZO. The triplet excited state has higher energy than excited singlet states, and although $s^{*} / s^{* *}$ states seem to be much more likely to occur; the three states would possibly be reached by irradiation during experimental measurements. Obviously, the decay process involves more than just two electrons, but in our model, two electrons per cell were used. The environment was considered during the simulations and became the model which is near the actual process as much as possible.

Finally and importantly, we have explored high cubic and tetragonal symmetries, but more excited electronic states can also be found with disordered structures belonging to orthorhombic, Amm2, and rhombohedral, R3m, symmetries. On the other hand, in this study, excited states have been localized and characterized at the DFT level. This very strong restriction can be assumed to be a limiting case. The theoretical description of physical/chemical processes that take place in excited electronic states needs a balanced method to describe all the electronic states involved (including both statistical and dynamical effects) to quantitatively assess these states. These improvements will be included in future research.

\section{CONCLUSIONS}

In summary, to better comprehend the BZO cathodoluminescence phenomena, first-principles calculations were able to localize and characterize the ground singlet and excited singlet and triplet electronic states for cubic and pseudo-tetragonal structures; singlet $\left(s, s^{* *}\right)$ and triplet $\left(t^{* *}\right)$ configurations were built on zirconium six off-center (structural defects) over $\Gamma$-point in reciprocal space. Density of states reports a predominance of $4 \mathrm{~d}_{\mathrm{z}^{2}}(\mathrm{Zr})$ orbital at $\mathrm{CB}$ with an important contribution of $4 \mathrm{~d}_{\mathrm{xz}}(\mathrm{Zr})$ and $2 \mathrm{p}_{\mathrm{z}} \mathrm{O}$ orbital in the VB for $s^{*}, s^{* *}$ and mainly to $t^{* *}$, given an insight about the strong structural polarization in $z$-direction, which was not observed to $s$ structure. The consistency between theoretical and experimental results suggests that polarized electronic triplet excited states could be the main responsible of CL behavior. This assertion is based on the Raman theoretical frequencies obtained for $t^{* *}$ configuration, where $\mathrm{Zr}$ atoms stretch asymmetrically with regard to two neighboring $\mathrm{O}$ atoms along the $z$ direction, characterized by $639 \mathrm{~cm}^{-1}(A)$ vibrational mode. The strategy developed here can also be further extended to other metal oxide materials.

\section{ACKNOWLEDGMENTS}

The authors dedicate this paper to the memory of Professor Purificación Escribano. They appreciate the support of the following Brazilian research financing institutions: CAPES, FAPESP/CEPID 98/14324-0, CNPq, and FAPESP. This research was partially developed at LNLS-National Laboratory of Synchrotron Light in the LME/LNNano/ CNPEM. This work was also supported by Generalitat Valenciana for Prometeo/2009/053 Project, Ministerio de Ciencia e Innovación for Project CTQ2009-14541-C02, Spanish MALTA-Consolider Ingenio 2010 Program (Project CSD2007-00045), Programa de Cooperación Científica con Iberoamerica (Brazil), Ministerio de Educación (PHB20090065-PC). A.B. acknowledges a research grant from Generalitat Valenciana BEST/2011. The authors are also grateful to Rorivaldo Camargo and Madalena Tursi for technical contributions.

${ }^{1}$ P. W. Anderson, Phys. Rev. 109(5), 1492-1505 (1958).

${ }^{2}$ F. Farges, G. E. Brown, and J. J. Rehr, Phys. Rev. B 56(4), 1809-1819 (1997).

${ }^{3}$ P. A. Lee and T. V. Ramakrishnan, Rev. Mod. Phys. 57(2), 287-337 (1985).

${ }^{4}$ F. Urbach, Phys. Rev. 92(5), 1324 (1953). 
${ }^{5}$ L. González, D. Escudero, and L. Serrano-Andrés, ChemPhysChem 13(1), 28-51 (2012).

${ }^{6}$ F. Boschini, A. Rulmont, R. Cloots, and R. Moreno, Ceram. Int. 35(3), 1007-1013 (2009).

${ }^{7}$ J. Huang, L. Zhou, Z. Wang, Y. Lan, Z. Tong, F. Gong, J. Sun, and L. Li, J. Alloys Compd. 487(1-2), L5-L7 (2009).

${ }^{8}$ H. Iwahara, Y. Asakura, K. Katahira, and M. Tanaka, Solid State Ionics 168(3-4), 299-310 (2004).

${ }^{9}$ K. D. Kreuer, Solid State Ionics 125(1-4), 285-302 (1999).

${ }^{10}$ H. J. Zhou, Y. B. Mao, and S. S. Wong, J. Mater. Chem. 17(17), 1707-1713 (2007).

${ }^{11}$ Z. Dong, T. Ye, Y. Zhao, J. Yu, F. Wang, L. Zhang, X. Wang, and S. Guo, J. Mater. Chem. 21(16), 5978-5984 (2011).

${ }^{12}$ E. A. Carter, Science 321(5890), 800-803 (2008).

${ }^{13}$ A. Bilic and J. D. Gale, Phys. Rev. B 79(17), 174107 (2009).

${ }^{14}$ J. W. Bennett, I. Grinberg, and A. M. Rappe, Phys. Rev. B 73(18), 180102 (2006).

${ }^{15}$ A. R. Akbarzadeh, I. Kornev, C. Malibert, L. Bellaiche, and J. M. Kiat, Phys. Rev. B 72(20), 205104 (2005).

${ }^{16}$ M. E. Bjorketun, P. G. Sundell, and G. Wahnstrom, Faraday Discuss. 134, 247-265 (2007)

${ }^{17}$ P. G. Sundell, M. E. Bjorketun, and G. Wahnstrom, Phys. Rev. B 73(10), 104112 (2006).

${ }^{18}$ R. A. Evarestov, Phys. Rev. B 83(1), 014105 (2011).

${ }^{19}$ M. F. C. Gurgel, M. L. Moreira, E. C. Paris, J. W. M. Espinosa, P. S. Pizani, J. A. Varela, and E. Longo, Int. J. Quantum Chem. 111(3), 694-701 (2011)

${ }^{20}$ R. Khenata, M. Sahnoun, H. Baltache, M. Rérat, A. H. Rashek, N. Illes, and B. Bouhafs, Solid State Commun. 136(2), 120-125 (2005).

${ }^{21}$ C. Zhu, K. Xia, G. R. Qian, C. L. Lu, W. Z. Luo, K. F. Wang, and J. M. Liu, J. Appl. Phys. 105(4), 044110-044118 (2009).

${ }^{22}$ P. K. Davies, H. Wu, A. Y. Borisevich, I. E. Molodetsky, and L. Farber, Annu. Rev. Mater. Res. 38(1), 369-401 (2008).

${ }^{23}$ L. Gracia, J. Andres, V. M. Longo, J. A. Varela, and E. Longo, Chem. Phys. Lett. 493(1-3), 141-146 (2010).

${ }^{24}$ E. Orhan, F. M. Pontes, C. D. Pinheiro, T. M. Boschi, E. R. Leite, P. S. Pizani, A. Beltran, J. Andres, J. A. Varela, and E. Longo, J. Solid State Chem. 177(11), 3879-3885 (2004).

${ }^{25}$ E. Orhan, J. A. Varela, A. Zenatti, M. F. C. Gurgel, F. M. Pontes, E. R. Leite, E. Longo, P. S. Pizani, A. Beltran, and J. Andres, Phys. Rev. B 71(8), 085113 (2005).

${ }^{26}$ M. L. Moreira, P. G. C. Buzolin, V. M. Longo, N. H. Nicoleti, J. R. Sambrano, M. S. Li, J. A. Varela, and E. Longo, J. Phys. Chem. A 115(17), 4482-4490 (2011).

${ }^{27}$ V. Longo, L. Cavalcante, M. Costa, M. Moreira, A. de Figueiredo, J. Andrés, J. Varela, and E. Longo, Theor. Chem. Acc. 124(5), 385-394 (2009).
${ }^{28}$ V. M. Longo, M. D. S. Costa, A. Z. Simoes, I. L. V. Rosa, C. O. P. Santos, J. Andres, E. Longo, and J. A. Varela, Phys. Chem. Chem. Phys. 12(27), 7566-7579 (2010)

${ }^{29}$ M. L. Moreira, E. C. Paris, G. S. do Nascimento, V. M. Longo, J. R. Sambrano, V. R. Mastelaro, M. I. B. Bernardi, J. Andres, J. A. Varela, and E. Longo, Acta Mater. 57(17), 5174-5185 (2009).

${ }^{30}$ M. L. Moreira, D. P. Volanti, J. Andrés, P. J. R. Montes, M. E. G. Valerio, J. A. Varela, and E. Longo, Scr. Mater. 64(2), 118-121 (2011).

${ }^{31}$ R. Borja-Urby, L. A. Díaz-Torres, P. Salas, E. Moctezuma, M. Vega, and C. Ângeles-Chávez, Mater. Sci. Eng., B 176(17), 1382-1387 (2011).

${ }^{32}$ L. Gracia, V. M. Longo, L. S. Cavalcante, A. Beltran, W. Avansi, M. S. Li, V. R. Mastelaro, J. A. Varela, E. Longo, and J. Andres, J. Appl. Phys. 110(4), 043501-043511 (2011).

${ }^{33}$ J. Götze, Mater. Charact. 60(7), 594-602 (2009).

${ }^{34}$ N. K. Reddy, M. Devika, N. Shpaisman, M. Ben-Ishai, and F. Patolsky, J. Mater. Chem. 21(11), 3858-3864 (2011).

${ }^{35}$ M. R. Gartia, Y. Chen, and G. L. G. Liu, Appl. Phys. Lett. 99(15), 151902 (2011).

${ }^{36}$ V. R. S. Dosevi, C. Roetti, R. Orlando, C. M. Zicovich-Wilson, F. Pascale, B. Civalleri, K. Doll, N. M. Harrison, I. J. Bush, P. H. D'Arco, and M. Llunell, CRYSTALO9 User's Manual (University of Torino, Torino, 2009).

${ }^{37}$ A. D. Becke, J. Chem. Phys. 98(7), 5648-5652 (1993).

${ }^{38}$ C. T. Lee, W. T. Yang, and R. G. Parr, Phys. Rev. B 37(2), 785-789 (1988).

${ }^{39}$ I. Gadaczek, K. J. Hintze, and T. Bredow, Phys. Chem. Chem. Phys. 14(2), 741-750 (2012).

${ }^{40}$ M. L. Moreira, J. Andres, V. R. Mastelaro, J. A. Varela, and E. Longo, Cryst. Eng. Comm. 13(19), 5818-5824 (2011).

${ }^{41}$ M. L. Moreira, J. Andres, J. A. Varela, and E. Longo, Cryst. Growth Des. 9(2), 833-839 (2009).

${ }^{42}$ R. Leonelli and J. L. Brebner, Phys. Rev. B 33(12), 8649-8656 (1986).

${ }^{43}$ J. Yu, J. L. Sun, J. H. Chu, and D. Y. Tang, Appl. Phys. Lett. 77(18), 2807-2809 (2000).

${ }^{44}$ K. P. Balashev, Koord. Khim. 15(1), 116-121 (1989).

${ }^{45}$ L. S. Cavalcante, V. M. Longo, M. Zampieri, J. W. M. Espinosa, P. S. Pizani, J. R. Sambrano, J. A. Varela, E. Longo, M. L. Simoes, and C. A. Paskocimas, J. Appl. Phys. 103(6), 063527 (2008).

${ }^{46}$ J. R. Ferraro, K. Nakamoto, and C. W. Brown, Introductory Raman Spectroscopy, 2nd ed. (Academic Press, Amsterdam, 2003).

${ }^{47}$ P. S. Dobal, A. Dixit, R. S. Katiyar, Z. Yu, R. Guo, and A. S. Bhalla, J. Appl. Phys. 89(12), 8085-8091 (2001).

${ }^{48}$ R. Borja-Urby, L. A. Diaz-Torres, P. Salas, M. Vega-Gonzalez, and C. Angeles-Chavez, Mater. Sci. Eng., B 174(1-3), 169-173 (2010).

${ }^{49}$ Y. V. B. de Santana, C. W. Raubach, M. M. Ferrer, F. La Porta, J. R. Sambrano, V. M. Longo, E. R. Leite, and E. Longo, J. Appl. Phys. 110(12), $123507(2011)$ 